\title{
La « question des races » dans un cadre administratif républicain : la création de la Commission nationale pour les études des relations interethniques
}

The "Race Issue" within a Republican Administrative Framework: The Creation of the 'Commission nationale pour les Études des Relations interethniques'

\section{Narguesse Keyhani}

\section{(2) OpenEdition}

Journals

Édition électronique

URL : http://journals.openedition.org/conflits/19544

DOI : $10.4000 /$ conflits. 19544

ISSN : $1777-5345$

Éditeur :

CCLS - Centre d'études sur les conflits lilberté et sécurité, L'Harmattan

Édition imprimée

Date de publication : 30 octobre 2017

Pagination : 61-76

ISBN : 978-2-343-13540-3

ISSN : 1157-996X

\section{Référence électronique}

Narguesse Keyhani, « La « question des races » dans un cadre administratif républicain : la création de la Commission nationale pour les études des relations interethniques », Cultures \& Conflits [En ligne], 107 | automne 2017, mis en ligne le 30 octobre 2019, consulté le 30 mars 2021. URL : http:// journals.openedition.org/conflits/19544; DOI : https://doi.org/10.4000/conflits.19544 


\section{La « question des races» dans un cadre administratif républicain : la création de la Commission nationale pour les études des relations interethniques 1}

\section{Narguesse KEYHANI}

Narguesse Keyhani est post-doctorante à l'ISP où elle participe à une enquête collective sur le traitement judiciaire des infractions racistes (JUSTIRACE / GIPJustice). Ses enquêtes (doctorale et en cours) portent sur la sociologie de l'action publique et les mobilisations contre le racisme et les discriminations.

« - $\begin{aligned} & \text { orce est de créditer l'immigré d'une culture » : c'est ainsi } \\ & \text { qu'Abdelmalek Sayad commente ironiquement l'inflation des usages }\end{aligned}$ de la notion de culture dans les discours politiques, administratifs, mais aussi militants sur les travailleurs immigrés à la fin des années 1970 2. Cette multiplication de discours sur la culture des migrants ne peut alors manquer d'interroger le sociologue de la double absence tant elle contraste avec la séquence historique qui précède. Comme l'ont montré de nombreux sociologues et socio-historiens, le cadrage dominant de ces questions jusqu'aux années 1970 est aveugle aux différences autres que celles qui découlent de la nationalité. Au sortir de la Seconde Guerre mondiale, un cadre légal excluant toute référence à la culture ou à l'ethnie s'est imposé dans l'appareil d'État ordonnant une appréhension du migrant à travers son statut de non national ou de travailleur. Cette exclusion n'est pas allée de soi, mais avec les ordonnances du 19 octobre et du 2 novembre 1945, ce sont finalement les hauts fonctionnaires issus de la Résistance qui parviennent à imposer un cadre légal refusant un cadrage ethnicisant ou culturaliste ${ }^{3}$.

1. Je remercie les évaluateurs et Sylvain Laurens pour leurs commentaires sur les versions précédentes de cet article.

2. Sayad A., Les Usages sociaux de la culture des immigrés, Paris, CIEMM, 1978.

3. Weil P., « Racisme et discrimination dans la politique française de l'immigration : 19381945/1974-1985 ", in Liberté, égalité, discriminations. L'identité nationale” au regard de l'histoire, Paris, Gallimard-Folio Histoire, 2008, pp. 23-88; Spire A., Étrangers à la carte : l'administration de l'immigration en France: 1945-1975, Paris, B. Grasset, 2005. 
La socio-histoire des pratiques administratives a nuancé la description de ce cadre légal officiellement "color-blind » : la reconstitution de l'entendement administratif et des pratiques des fonctionnaires de guichet a mis en évidence que l'octroi de titres de séjour ${ }^{4}$, la mise en ouvre de politiques municipales de logement ${ }^{5}$, ou encore la gestion des foyers SONACOTRA 6 pouvaient prendre en compte les origines des travailleurs. Mais c'est en s'éloignant des débats politiques et administratifs et du cadre législatif et en observant les pratiques administratives au niveau local que l'écart entre cadre légal et pratiques administratives a pu être souligné.

Jusqu'aux années 1970, toute l'administration abordait-elle pour autant la question des migrants sans recourir aux terminologies racialistes qui prévalent avant-guerre ${ }^{7}$ ou aux références culturalistes qui imprègnent l'administration étasunienne ou certaines organisations internationales ? En explorant la naissance, en France, d'un intérêt savant pour les relations interculturelles et interethniques dès les années 1960, l'article propose de qualifier cette "force " qu'évoque Abdelmalek Sayad et qui semble surgir subitement à la fin des années 1970. Il s'intéresse pour cela aux acteurs qui ont promu ces savoirs, à leurs argumentaires et au cadre institutionnel qu'ils ont institué dès le milieu de la décennie précédente.

Le 19 janvier 1966, un arrêté de la Direction de l'enseignement supérieur du ministre Christian Fouchet institue en effet la Commission nationale pour les études des relations interethniques (CNEI) et définit les missions du Centre d'études des relations interethniques $\left(\right.$ CERIN $\left.{ }^{8}\right)$ installé à la Faculté des Lettres et sciences humaines de l'université de Nice. Le Centre a " pour objet d'étudier les causes et les conditions rendant fructueux ou nocifs les contacts entre ethnies 9 » pour armer la politique française de coopération. Au cours des années 1970, un petit groupe de sociologues y conduira des enquêtes et assurera des formations destinées tant aux coopérants qu'aux formateurs de travailleurs immigrés en France, se réclamant ainsi du domaine d'études et d'actions de la sociologie des relations interculturelles et interethniques. Il est également attendu de ces savoirs de contribuer au renouvellement du regard posé sur la condition des immigrés en France. En considérant la société

4. Spire A., Étrangers à la carte : l'administration de l'immigration en France : 1945-1975, op. cit.

5. De Barros F., «Contours d'un réseau administratif “algérien” et construction d'une compétence en "affaires musulmanes" ", Politix, vol. 4, n 76, 2006, pp. 97-117.

6. Hmed C., «L’encadrement des étrangers “isolés” par le logement social (1950-1980) », Genèses, vol. 3, n 72, 2008, pp. 63-81.

7. Weil P., «Racisme et discrimination dans la politique française de l'immigration : 19381945/1974-1985 », op. cit. ; Larbiou B., «René Martial, 1873-1955. De l’hygiénisme à la raciologie, une trajectoire possible », Genèses, vol. 60, n 3, 2005, pp. 98-120.

8. En 1970, le Centre d'études est renommé Institut d'études et de recherches interethniques et interculturelles (IDERIC). Dans les années 1960-1970, « interculturel » et « interethnique » semblent interchangeables pour les hauts fonctionnaires.

9. Article 1 de l'arrêté du 19 janvier 1966 de la Direction de l'enseignement supérieur créant la Commission nationale pour les études des relations interethniques. 
comme constituée de groupes caractérisés par leurs cultures, en promouvant l'étude des relations interculturelles et interethniques, ces sociologues et ces agents administratifs qualifient en de nouveaux termes les rapports sociaux qui ne pourraient être réduits à des rapports entre classes.

Dès les années 1960, cette Commission nationale pour les études des relations interethniques développe un discours sur l'immigration où les faits culturels et ethniques tiennent une place centrale et explicite. Certes, elle ne parvient pas à imposer ses analyses et sa terminologie dans l'appareil administratif et il faudra attendre encore quinze ans pour qu'une action publique s'institutionnalise autour d'un problème des relations interculturelles ${ }^{10}$. Mais ce cas donne à voir la consolidation d'un discours sur les cultures des immigrés qui n'est annexé ni à l'objectif d'assimilation, ni à celui de la description des discriminations, et qui est porté par des hauts fonctionnaires français dès les années 1960.

En s'intéressant à l'émergence d'un besoin de savoirs sur les relations interethniques et interculturelles à travers les trajectoires d'une poignée de hauts fonctionnaires, l'article éclaire de 1960 au début des années 1970 un épisode de la problématisation de la présence immigrée en France que l'analyse des pratiques administratives au guichet ou des diverses politiques de lutte contre les inégalités a laissé dans l'ombre. Cette histoire a été jusqu'ici peu investie car elle suppose d'articuler des dynamiques administratives transnationales avec l'analyse de l'administration française. L'investissement des études interethniques dans les années 1960 par des hauts fonctionnaires éloignés par leurs fonctions de la politique de l'immigration ne peut se comprendre qu'en considérant les transformations de la position de la France sur la scène internationale depuis les années 1950. Elles sont marquées, d'une part, par la création de l'Organisation des Nations Unies (ONU), de celle dédiée à l'éducation, la science et la culture (UNESCO) et par la présence française en leur sein et, d'autre part, par l'éclatement de l'empire colonial qui aboutit à l'indépendance des colonies françaises et à l'émergence d'une politique de Coopération. Les hauts fonctionnaires qui défendent en France le développement des études sur les relations interethniques et interculturelles sont bien souvent des universitaires qui ont par ailleurs mené une carrière à l'intersection des organisations onusiennes et de la politique scientifique française. Leurs trajectoires entre ces différents espaces peuvent être lues au prisme des transformations des relations extérieures de la France. Leur mobilité entre ces différents espaces qui renvoient aussi à différents secteurs d'action publique (politique scientifique onusienne, politique française de coopération scientifique), permet de comprendre comment émerge l'idée que la France doit être un lieu de production de savoirs sur les relations interethniques, et de saisir les

10. Keyhani N., Les "relations interculturelles" : trajectoire sociale d'une catégorie réformatrice, Thèse de doctorat, ENS Cachan, Cachan, 2014. 
ressources qu'ils mobilisent pour formuler et justifier ce besoin de savoirs.

Une littérature conséquente existe sur les rapports entre pouvoirs publics, sciences et sciences sociales. Si la question de l'expertise y tient une place centrale, d'autres travaux s'intéressent aux conditions institutionnelles et historiques de développement des sciences sociales, sans porter directement sur la mobilisation de savants et de savoirs dans les processus de décision. L'article s'inscrit dans cette deuxième perspective en décrivant comment de hauts fonctionnaires deviennent des « entrepreneurs de recherche 11 » dont les propriétés biographiques, expériences, réseaux et ressources sont opportunément mobilisés dans le cadre de leur circulation entre différents secteurs d'action publique et entre scènes internationale et nationale. L'article prend acte de l'un des apports des travaux sur le rôle des institutions internationales dans l'édification des politiques publiques nationales : les circulations de personnes, d'idées, de normes, de problèmes publics entre différents espaces ne sont jamais des importations et transferts purs et simples ${ }^{12}$. Les institutions internationales s'apparentent plutôt à des espaces de consolidation de réseaux d'expertise qui peuvent être stratégiquement mobilisés sur les scènes nationales ${ }^{13}$.

La description de la naissance d'un objet de recherche en train de se constituer expose au problème des sources, accru par la marginalité qui caractérise la Commission nationale. Ce problème a pu être contourné par la mobilisation de sources éparses. Le Fonds Charles Morazé, historien et haut fonctionnaire impliqué dans la CNEI, a permis de reconstituer les étapes de la création de la Commission et les réflexions qui y ont été menées. Les interventions publiques de ces hauts fonctionnaires (articles de presse, articles dans des revues spécialisées, intervention dans des colloques) ont permis de documenter l'élaboration d'un besoin de savoirs. Ces données ont été croisées avec les trajectoires de ces hauts fonctionnaires, reconstituées grâce aux multiples biographies établies par le CNRS ou les centres de recherche dans lesquels ils étaient impliqués. L'article montre comment le passage de ces hauts fonctionnaires par les institutions onusiennes a favorisé la formulation d'un besoin de savoirs sur les relations interethniques en France, avant de montrer comment se construit l'analogie entre situations d'immigration et de coopération, au cour de ce programme de recherche.

11. Bezes P., Chauvière M., Chevallier J., De Montricher N. et F. Ocqueteau (dir.), L'État à l'épreuve des sciences sociales : la fonction recherche dans les administrations sous la Ve République, Paris, La Découverte, 2005, p. 13.

12. Saunier P.-Y., "Circulations, connexions et espaces transnationaux », Genèses, vol. 4, n 57, 2004, pp. 110-126.

13. Kott S., "Une “communauté épistémique” du social ?", Genèses, vol. 2, n 71, 2008, pp. 2646. 


\section{La doxa antiraciste des institutions onusiennes}

Ce sont quatre hauts fonctionnaires internationaux, par ailleurs enseignants-chercheurs, qui encadrent la Commission nationale et le Centre d'études interethniques. Henri Laugier est nommé à la CNEI, Georges Fisher en devient le secrétaire général, Charles Morazé le président, tandis que Pierre Bessaignet prend la direction du Centre d'études. De formations disciplinaires différentes, parfois éloignées, ils partagent une expérience professionnelle dans le cadre des institutions onusiennes dans les années 1950, où ils ont souvent tenu des responsabilités relatives à la politique scientifique, qu'ils ont par ailleurs investie sur la scène nationale, avant, parallèlement ou après leur entrée dans ces institutions. Lorsqu'ils défendent dans les années 1960 la création en France d'un centre d'études interethniques, cette socialisation professionnelle internationale les incite à mobiliser des références et grilles de lecture portées initialement par ces institutions dans les années 1950.

Car dès leur création, l'ONU comme l'UNESCO sont le théâtre de débats et de programmes de recherche sur la « question des races » et du racisme ${ }^{14}$. Ces programmes sont intimement liés au contexte de naissance de ces institutions, marqué par la fin de la Seconde Guerre mondiale et l'extermination des Juifs d'Europe. La forme prise par ces débats s'éclaire également par l'enrôlement de chercheurs, qui permet de construire un discours d'invalidation scientifique de la notion biologique de race. Après la «Déclaration sur la Race » de 1949 qui pose les bases d'une doctrine antiraciste, la «Division pour l'étude des questions de race " du Département des sciences sociales de l'UNESCO, dirigé par l'anthropologue Alfred Métraux, commande une série d'études pour étayer cette doctrine à partir de travaux de différentes disciplines ${ }^{15}$. C'est dans le cadre du projet de publication « la question raciale devant la science moderne » que ces études sont publiées dans la première moitié des années 1950, notamment l'ouvrage de Claude Lévi-Strauss Race et histoire en 1952 16. D’autres monographies sont commanditées pour illustrer « les situations raciales heureuses » à travers le monde, auxquelles participe notamment Michel Leiris ${ }^{17}$. Ces institutions développent aussi des programmes de recherche sur l'histoire et le dialogue entre «civilisations 18 ».

14. Maurel C., «La question des races », Gradbiva, n 5, 2007, pp. 114-131 ; Brattain M., « Race, Racism, and Antiracism : UNESCO and the Politics of Presenting Science to the Postwar Public ", The American Historical Review, vol. 112, n 5, 2007, pp. 1386-1413.

15. Maurel C., «La question des races ", op. cit.

16. Lévi-Strauss C., Race et histoire, Paris, Unesco, 1952.

17. Leiris M., Contacts de civilisations en Martinique et en Guadeloupe, Paris, UnescoGallimard, 1955.

18. Sur les réseaux intellectuels et transnationaux qui constituent l'arrière-plan de cette entreprise collective de rédaction d'une encyclopédie et à laquelle participent notamment Bertrand Russel et Arnold Toynbee, voir Maurel C., « L’Unesco : une plate-forme pour les circulations transnationales de savoirs et d'idées (1945-1980) », Histoire@Politique, vol. 3, n 15, 2011, pp. $42-59$. 
Marqués par le sceau de «l'universalisme » et de la "paix entre les nations », ces discours se déploient alors que le mouvement des non-alignés prend forme entre les décolonisations et la guerre froide. Les discours sur les races, le racisme et la paix se développent dans un cadre où les problématiques du tiersmondisme et de l'équilibre entre les nations sont également présentes.

Ce contexte permet de comprendre, d'une part, quelles sont les ressources discursives dont disposent les défenseurs des études sur les relations interethniques et interculturelles en France et, d'autre part, selon quelles logiques et pour quelles finalités elles sont mobilisées opportunément sur la scène nationale. C'est bien à l'intersection des scènes internationale et nationale qu'un besoin de savoirs spécifiques est promu et défini en des termes conciliables avec la définition d'un rôle spécial revenant à la France.

Des trajectoires à la croisée de la scène internationale et de la politique scientifique française

La carrière professionnelle d'Henri Laugier suit deux voies : celle de l'enseignement et de la recherche dans un premier temps, celle de la fonction publique nationale et internationale par la suite. Né en $1888{ }^{19}$ dans une famille républicaine, proche du socialisme jaurésien, sa carrière est d'abord scientifique. Après une thèse de médecine en 1913 et une thèse de physiologie en 1921, il est nommé en 1929 professeur de physiologie au Centre national des Arts et Métiers. Pendant ses études, il est secrétaire du groupe des Étudiants socialistes au quartier latin. Il rejoint également les Compagnons de l'Université Nouvelle, cercle qui milite pour l'École unique ${ }^{20}$.

En octobre 1939, il est nommé premier directeur du CNRS et le reste jusqu'à l'été 1940, date à laquelle il est démis de ses fonctions par le gouvernement de Vichy. La guerre le pousse à s'exiler au Canada, où il obtient, grâce à la fondation Rockefeller, la chaire de physiologie de la faculté de médecine de l'Université de Montréal. Après son investissement dans l'accueil des scientifiques français réfugiés aux États-Unis, son implication auprès des intellectuels et universitaires qui s’y sont installés (comme Paul Rivet ou Claude LéviStrauss) et dans la propagande de la France libre en Amérique du nord, Charles de Gaulle le nomme en 1943 recteur de la première université fran-

19. Ces éléments biographiques sont tirés de Fischer G., «Henri Laugier. 1888-1973 », Politique étrangère, $\mathrm{n}^{\circ}$ 1, 1973, pp. 99-102 ; Prost A., «Les origines des politiques de la recherche en France (1939-1958) ", Cabiers pour l'bistoire du CNRS 1939-1989, vol. 1, 1988 ; Collectif (dir.), Cahiers pour l'bistoire de la recherche, CNRS Éditions, Paris, 1995 ; Dosso D., «Les scientifiques français réfugiés en Amérique et la France Libre », Matériaux pour l'histoire de notre temps, vol. 60, n 1, 2000, pp. 34-40; Racine, N., " Paul Rivet, Vichy et la France libre 1940-1944 », Histoire@Politique, vol. 1, n 1, 2010.

20. C'est grâce à ces réseaux et à son séjour à la fondation Thiers de 1919 à 1921 qu'il noue ses premiers contacts avec de futurs hauts fonctionnaires du secteur de l'éducation dont on peut présumer qu'ils ont favorisé ses liens avec le ministre Christian Fouchet. 
çaise libre à Alger. Il entame à la Libération une carrière de fonctionnaire international. Il est le premier secrétaire général adjoint des Nations Unies de 1946 à 1951, responsable de la section des Affaires sociales ${ }^{21}$. En 1953, il est désigné par l'assemblée générale de l'ONU comme membre de la Commission pour l'étude de la situation raciale en Afrique du Sud. Il est par ailleurs membre du Conseil exécutif de l’UNESCO de 1952 à 1958. Il retrouve parallèlement la politique scientifique française en devenant en 1953 président de la Commission de la Recherche scientifique et technique du second Plan et en créant l'Institut du Développement économique et social en 1957. Il milite plus généralement pour la création d'un ministère pour la recherche scientifique et tire de ces expériences une grande familiarité avec la haute administration française et les institutions onusiennes.

Né en 1913, Charles Morazé 22 est historien de formation, agrégé et enseigne l'histoire économique et sociale à l'École libre des sciences politiques à partir de 1941. Boursier de la fondation Thiers en 1938-1939 et en 19411943, il enseigne à la Ivème section de l'École pratique des hautes études (EPHE) à partir de 1943. Avec Lucien Febvre et grâce à ses propres liens avec la fondation Rockefeller ${ }^{23}$, il fonde la vième section de l'EPHE dédiée aux sciences économiques et sociales. À partir de 1946, il codirige avec Fernand Braudel, Georges Friedmann et Lucien Febvre la revue des Annales. Ses premiers travaux relèvent de l'histoire économique et sociale et portent sur la bourgeoise française ${ }^{24}$. Au fil de sa carrière, ses objets de recherche se diversifient : il travaille sur les conditions culturelles du progrès scientifique dans le cadre de la Commission internationale pour une histoire du développement scientifique et culturel de l'humanité de l'UNESCO, qu'il a intégrée en 1950 et dont il est rapporteur jusqu'en 1961. C'est dans ce cadre qu'il contribue au projet d'encyclopédie historique Histoire du Développement Scientifique et Culturel de l'Humanité 25 lancé dès 1947, publié en 1968 et qu'il coordonne avec Julian Huxley et Lucien Febvre. Malgré la mobilisation d'un registre universaliste et d'égalité entre les peuples, ces travaux ne sont pas sans ambiguïtés dans le traitement qu'ils réservent aux civilisations des pays du Sud. Non seulement l'œuvre consacre une place majeure à l'histoire des civilisations occidentales, mais elle est par ailleurs parsemée de considérations laissant transparaître la croyance en une hiérarchie des civilisations et cultures 26 .

21. Stéphane Hessel rejoindra son cabinet.

22. Biographie établie à partir du site internet du Service des Archives de l'EHESS et de « Charles Morazé et les études interethniques ». Non daté. Fonds Charles Morazé : 8D231.

23. Revel J., Wachtel N. et M. Augé, Une école pour les sciences sociales : de la VIe Section à l'École des hautes études en sciences sociales, Paris, Éd. de l'EHESS, 1996.

24. Morazé C., La France bourgeoise, XVIII $-X X^{e}$ siècles, Paris, A. Colin, 1946 ; Les bourgeois conquérants : XIXe siècle, Paris, Armand Colin, 1957.

25. Gottschalk L.R. et C. Morazé, Histoire du développement culturel et scientifique de l'bumanité. Volume V, Le XIXe siècle, Paris, R. Laffont, 1969.

26. Maurel C., «L'UNESCO entre européocentrisme et universalisme (1945-1974) », Les cabiers 
Charles Morazé n'est pas seulement convoqué à l'UNESCO en tant qu'historien. De 1947 à 1968, il est membre titulaire de la Commission nationale française pour l'UNESCO. Il est en effet proche du général de Gaulle et des ministres Pierre Mendès France et Christian Fouchet dont il a été conseiller technique de juin 1954 à février 1955, alors qu'il était ministre chargé des Affaires marocaines et tunisiennes, puis auprès de Charles de Gaulle en 19581959, alors président du Conseil. Il retrouve Christian Fouchet en devenant conseiller auprès du Directeur des enseignements supérieurs en 1965.

Au cours des années 1950, ces hauts fonctionnaires se situent à la croisée de réseaux de sociabilité scientifique (notamment du fait de leur position centrale dans le cadre de la politique scientifique) et politiques (réseaux de la France libre, plus généralement gaullistes, réseaux socialistes), sur les scènes nationale et internationale.

\section{Le cas sud-africain, le "rôle de la France » et les études interethniques}

Lorsqu'au milieu des années 1960, Henri Laugier s’investit dans la Commission nationale pour les études des relations interethniques, c'est au terme d'un parcours qui l'a éloigné du monde de la recherche. Il est désormais un habitué des institutions onusiennes. Il est entré au Conseil exécutif de l'UNESCO en 1952, c'est-à-dire dès les premières publications de l'institution sur la question des races et du racisme. Quelques années plus tard, les interventions publiques de Henri Laugier sont clairement marquées par sa familiarisation, au début des années 1950, avec ces questions telles qu'elles ont été formulées au sein de l'UNESCO. En février 1960, il prend part à une controverse française sur la situation sud-africaine dans la revue Droit et Liberté 27 . Dans un article titré "Alors que tout condamne le racisme... », il répond au général Lionel Max Chassin, militant de l'Algérie française et défenseur du régime sud-africain. Rappelant le rôle de la France dans la rédaction de la Déclaration universelle des droits de l'homme à laquelle il a participé, liant l'universalisme des textes déclaratifs des organisations onusiennes avec le « rôle de la France », Henri Laugier mobilise les études menées par

Irice, vol. 1, n 9, 2012, pp. 61-72. Pour une critique de la réduction de l'antiracisme de l'UNESCO au racisme biologique, voir Lentin A., Racism and anti-racism in Europe, London, Pluto Press, 2004, pp. 72-112. Sur la dimension controversée de la mission éducative de l'UNESCO, voir Wong E.L., « Intergovernmental organizations (IGOs) », in Irie A., Saunier P.-Y. (dir.), The Palgrave dictionary of transnational bistory, Basingstoke, Hampshire, 2009, pp. 555-561.

27. Laugier H., Du civisme national au civisme international, Gap, Éd. Ophrys, 1972. L'ouvrage réunit de nombreux articles du haut fonctionnaire. Les courts articles évoqués ici sont rassemblés dans une partie intitulée : "Contre le racisme ». Elle réunit trois articles publiés dans la première moitié des années 1960 : «Alors que tout condamne le racisme... », in Du civisme national au civisme international, Gap, Éd. Ophrys, 1972, pp. 508-510 ; "Afrique du sud problème international ", in Du civisme national au civisme international, Gap, Éd. Ophrys, 1972, pp. 511-515 ; "Pour un institut interethnique ", Du civisme national au civisme international, Gap, Éd. Ophrys, 1972, pp. 516-518. 
l'UNESCO publiées quelques années plus tôt pour saper les fondements scientifiques de la discrimination raciale. Dans un autre article, «Afrique du Sud, problème international », publié le 3 mai 1960 dans le journal Combat où il a des entrées depuis la Seconde Guerre mondiale, il appelle à une intervention extérieure en Afrique du Sud. Lorsque Henri Laugier intervient en 1960 sur la question du racisme et des relations raciales, c'est donc à travers le cas sud-africain qui, depuis le milieu des années 1950, focalise l'attention du « comité d'experts sur la question des races »du Département de sciences sociales de l'UNESCO. Si certains pays membres avaient souhaité aborder la question raciale dans différents pays, notamment aux États-Unis, dans les colonies françaises et britanniques, ils ne parviennent pas à imposer l'extension de cet enjeu aux démocraties libérales ${ }^{28}$.

C'est dans l'article « Pour un institut interethnique », publié le 26 septembre 1964 dans Combat ${ }^{29}$, qu'il évoque explicitement la situation française et la nécessité de développer les recherches sur les questions interethniques : «Les tensions entre races, et les discriminations qui sévissent dans le monde, posent des problèmes graves et en aggravation continue, dans un nombre croissant de pays. Au sein des États nouveaux, elles retardent l'indispensable intégration nationale [...]. Dans les pays développés, elles constituent un danger permanent plus ou moins aigu pour les structures mêmes de l'État. Elles rendent difficile ou compromettent l'entente et la compréhension entre les pays du TiersMonde et le nôtre, entente et compréhension qui sont d'une importance majeure pour la paix [...]. Il est souhaitable que les solutions à apporter à ces difficultés ne soient pas laissées exclusivement aux mains d'administrateurs ou d'hommes politiques, tous pleins d'une immense bonne volonté, mais de toute évidence insuffisamment informés sur le fond des choses, et sur les éléments historiques, géographiques, biologiques, psychologiques qui sont en jeu. Il est donc nécessaire que des mécanismes d'études, de recherches, de pensée objective soient mis en place pour fournir aux gouvernements toutes les informations et suggestions réfléchies, dont le besoin est évident 30 ».

Selon Henri Laugier, c'est l'état du monde et en particulier des relations entre pays développés et en voie de développement qui rend nécessaires de telles études, bien que les pays industrialisés soient aussi touchés par des tensions entre les groupes ethniques. S’inspirant des études commanditées par l'UNESCO sur « les relations raciales », il estime qu'en raison de l'expérience française de la « diversité », ce centre de portée internationale doit être installé

28. L'UNESCO commandite des études sur l'apartheid dès 1954. En 1967, elle publie d'ailleurs, sous l'impulsion de la commission onusienne pour l'étude de la situation sud-africaine, « un rapport sur les effets de la politique d'apartheid sur l'éducation, la science, la culture et l'information en Afrique du sud ». Maurel C., «La question des races », op. cit.

29. Morazé C., Un historien engagé. Mémoires, Paris, Fayard, 2007, pp. 341-352. C’est dans une partie intitulée «Le malheur des autres » et dans la section «Les laissés-pour-comptes» qu'est évoquée la création de la CNEI.

30. Laugier H., "Pour un institut interethnique », op. cit., p. 516. 
en France. S'il ne développe pas son analyse de cette réalité française, il évoque rapidement que la coexistence peut être problématique. Dix ans après les premiers travaux de l'UNESCO et les débats qui ont traversé l'institution sur l'extension de l'analyse des relations raciales aux démocraties libérales, au-delà du cas sud-africain, Henri Laugier reste ainsi discret sur les relations raciales dans les colonies et anciennes colonies françaises ${ }^{31}$.

Pour Henri Laugier, l' «Institut interethnique » doit s'inscrire dans le sillage des programmes de recherches initiées par l'UNESCO, comme il en existe déjà en Grande-Bretagne et aux États-Unis. Tous les enjeux de la création d'un tel institut sont ici résumés : le rôle moteur de la France dans la production de savoirs, la crainte d'être dépassé par les concurrents étasuniens et britanniques, l'enjeu des relations extérieures de la France et, enfin, l'articulation du nouveau champ de savoirs avec ceux de la Coopération et de l'immigration. Si Henri Laugier apparaît ainsi comme un « opérateur de circulation 32 » d'idées entre la scène internationale, où il a tenu diverses responsabilités de la Libération au dernier tiers des années 1950, et la scène nationale qu'il a rejointe en 1952 dans le cadre du Plan, il s'agit de les mobiliser en assurant le rayonnement de la France et d'assurer l'influence française dans le champ scientifique pensé ici à l'échelle internationale. Il s'agit aussi d'œuvrer à la paix entre les États. La meilleure connaissance des relations de « cohabitation » des différents groupes dans les pays industrialisés doit contribuer à une meilleure «compréhension » entre la France et les pays du Tiers Monde. Par ses interventions dans la presse, en puisant dans son expérience de haut fonctionnaire international et à partir du cas sud-africain, il construit une demande publique de savoirs sur les relations interethniques qui reformule les termes du programme onusien de recherche en fonction des intérêts des relations extérieures de la France avec les pays du «Tiers Monde ». Ces relations sont alors réorganisées autour de la coopération.

\section{Repenser les relations de la France avec le «Tiers Monde»}

La structuration de la politique de développement est intimement liée à l'éclatement de l'empire colonial. Le ministère de la Coopération créé en 1959 permet d'assurer aux autorités françaises leur influence sur leurs possessions en voie d'émancipation, autrement dit, malgré les indépendances ${ }^{33}$. La mise en scène de la fin des institutions coloniales (notamment du ministère de la France d'outre-mer, des lieux de formation des fonctionnaires coloniaux, etc.)

31. Julien Meimon rappelle d'ailleurs que la France fait l'objet de nombreuses critiques, au sein même des institutions onusiennes, pour sa gestion de la « crise algérienne ». Meimon J., "L'invention de l'aide française au développement. Discours, instruments et pratiques d'une dynamique hégémonique », Questions de Recherche-CERI, n² 21, 2007.

32. Kaluszynski M., Payre R., «Introduction », Savoirs de gouvernement : circulation(s), traduction(s), réception(s), Paris, Economica, 2013, pp. 7-19.

33. Meimon J., En quête de légitimité : le ministère de la Coopération (1959-1999), Thèse de doctorat, Université du droit et de la santé, Lille, 2005. 
dans la deuxième moitié des années 1950 s'accompagne de la création d'autres espaces et institutions autour de l'enjeu de « l'aide au développement » par la reconversion des agents coloniaux. C'est de la rencontre entre, d'une part, l'intérêt des élites politiques françaises à maintenir leur influence sur l'Afrique francophone afin de parer à la concurrence des autres puissances mondiales et, d'autre part, la nécessaire reconversion de milliers de fonctionnaires coloniaux, que naît une entreprise de justification de l'aide française au développement, sous les traits d'un projet altruiste, pacifiste et mâtiné de tiers-mondisme : la France apporte son « assistance technique » pour lutter contre les disparités croissantes entre nations, à la fois injustes et nuisibles à la paix. L'aide au développement apparait également comme un discours technicisé et dépolitisé. Il met en sourdine l'enjeu de l'influence française et son souci d'entretenir son leadership en Afrique francophone.

La politique de développement (ou de coopération) se décline dans les années 1960 en une diversité de discours à l'origine de l'idée d'un entremêlement des enjeux de développement et d'immigration. L'idée qu'il est possible de contribuer au développement à travers l'immigration repose sur une analyse des crises des sociétés "sous-développées » les imputant à l'exode rural, perçu comme un puissant facteur de déstabilisation. Les immigrés, par les transferts de capitaux et de savoir-faire, apparaissent alors comme susceptibles de contribuer au développement des sociétés d'émigration ${ }^{34}$. Dans les milieux de la coopération comme la "Commission d'étude de la politique de coopération », créée en 1963 et où se développent ces analyses, la formation est alors érigée en levier à privilégier dans cette perspective. À travers les prises de position des fondateurs de la CNEI sur la coopération, on peut déceler les linéaments de ce paradigme « développementaliste 35 » qui s'incarnera pleinement à travers les activités du Centre d'études.

\section{Le développement et la question de la formation}

Né en 1917 36, proche du syndicaliste et résistant Louis Saillant, George Fischer s'engage au sein de la Fédération syndicale mondiale (FSM ${ }^{37}$ ) dont il dirige le département économique et social. Il représente la FSM à l'ONU. Parallèlement à cette carrière internationale, il est chercheur en droit et science politique : maître de recherche au CNRS, il finira sa carrière à l'Institut des Sciences juridiques du Développement de Paris v. Ses sujets de

34. Dedieu J.-P., La parole immigrée : les migrants africains dans l'espace public en France, 19601995, Paris, Klincksieck, 2012.

35. Ibid., p. 113.

36. Ces données s'appuient sur Morazé C., «A l'IEDES : Georges Fischer », Tiers-Monde, vol. $15, \mathrm{n}^{\circ}$ 58, 1974, pp. 413-414.

37. Créée en 1945, la FSM se distingue de la Confédération internationale des syndicats libres, née en 1949 d'une scission de la FSM dans le contexte de guerre froide. Après la scission, la FSM constitue le pôle syndical revendiquant nettement un attachement au marxisme. 
recherche portent sur les décolonisations, les relations internationales et le développement ${ }^{38}$.

Proche d'Henri Laugier, probablement depuis leurs expériences aux Nations Unies 39, il crée avec lui en 1957 l'Institut du développement économique et social (IEDES) qu'il dirigera jusqu'en 197340 ainsi que la revue Tiers Monde née avec l'Institut. Après avoir quitté son poste à l'ONU dans les années 1950, Henri Laugier réinvestit la politique scientifique française, dans le sillage des transformations de la politique française en direction des anciennes colonies ou en voie de le devenir. D'ailleurs, l'IEDES comme la revue Tiers Monde répondent aux préoccupations du gouvernement français d'anticiper les décolonisations en cours en redéfinissant le cadre des relations de la France avec les futurs États indépendants, de former les élites de ces mêmes pays et d'actualiser les connaissances sur ces sociétés ${ }^{41}$. Dans ce contexte, Henri Laugier et Georges Fischer défendent une politique de coopération qui passe essentiellement par la formation des élites des pays en voie de développement.

En témoigne leur participation au colloque sur la recherche et l'enseignement dans les pays de la Communauté française organisé en décembre 1959 à Abidjan puis à Dakar par L'Association d'étude pour l'expansion de la recherche scientifique. Leur intervention « Pour une université internationale au service des pays sous-développés 42 » est publiée dans Tiers Monde en 1960. Les auteurs prennent part au débat sur l'opportunité de créer des universités dans les principaux centres urbains de la Communauté française. Selon les défenseurs de cette option, ces universités doivent se doter d'un personnel français issus de métropole et suivre le modèle des universités métropolitaines, par opposition aux structures universitaires dites « adaptées » aux pays ou territoires en question. Georges Fischer et Henri Laugier rejettent cette option et proposent plutôt un programme de formation des cadres autochtones dans une seule et même " université africaine ». La promotion de l'autonomie des cadres universitaires de ces pays par la formation doit primer sur le rôle de la France dans le maillage universitaire de ses anciennes colonies.

38. Fischer G., Un cas de décolonisation : les États-Unis et les Philippines, Paris, LGDJ, 1960 ; Syndicats et décolonisation, Paris, FSNP, 1961 ; Le parti travailliste et la décolonisation de l'Inde, Paris, F. Maspero, 1966.

39. Fischer G., "Henri Laugier : souvenirs. De l'ONU au Centre d'études des relations interethniques », 1995, coll. «CNRS Éditions ».

40. Date à laquelle il est remplacé par Stéphane Hessel, issu du monde de la coopération et qui s'investit dans les années 1970 dans la promotion des relations interculturelles.

41. Guichaoua A., " Parcours d'une institution : l'IEDES a 50 ans au terme de cinq décennies du développement », Tiers-Monde, vol. 3, n 191, 2007, pp. 647-659.

42. Fischer G. et $\mathrm{H}$. Laugier, « Pour une Université internationale au service des pays sous-développés », Tiers-Monde, vol. 1, n 1-2, 1960, pp. 17-26. 
Plusieurs points importants se dégagent de cette intervention. La dimension prospective et normative de l'exposé de cette doctrine du développement place les auteurs en conseillers des acteurs de la politique de coopération scientifique. Surtout, l'article loge au cour de la réflexion la question de la formation des élites indigènes des pays dits sous-développés. Ainsi, le futur secrétaire général de la CNEI et celui qui en porte le projet auprès du ministère de la Direction de l'Enseignement supérieur posent dès 1960 les termes selon lesquels la question des études sur les relations interethniques est formulée quelques années plus tard. Cette réflexion naît dans un cadre à la fois différent de celui de la Commission nationale et directement tributaire de la politique de coopération scientifique en train de prendre forme, alors que le ministère de la Coopération vient juste d'être créé : elle pose la question des conditions de transmission des savoirs et savoir-faire aux élites des pays en voie de développement.

Pour défendre l'autonomie des cadres universitaires africains, Henri Laugier et Georges Fischer évoquent le risque de renouer avec un expansionnisme français. Mais la question de l'influence française est explicite dans leur intervention : «Le seul "nationalisme" qui nous reste, nous espérons qu'on nous le pardonnera, est de souhaiter que la France prenne l'initiative de cette proposition et décide de conduire le combat pour en assurer la réalisation dans les milieux internationaux ; cette initiative correspond bien à la représentation que le monde, en majorité, se fait de la mission d'universalité intellectuelle et culturelle de la France. Elle serait aussi conforme à l'image que, avec Michelet, tant de Français se font de leur pays 43 ». La mise à distance du colonialisme à travers leur intervention s'inscrit ainsi dans la stratégie de justification de la politique de coopération, de mise en scène d'une rupture avec l'expérience coloniale et, dans le même temps, de préparation du maintien d'une influence française, exclusive, sur l'Afrique francophone ${ }^{44}$. C'est en ce sens que l'enjeu de la formation dans le cadre de la coopération sera au cœur des missions du Centre d'études sur les relations interethniques, lorsque quelques années plus tard, Henri Laugier mènera une campagne pour sa création, par l'entremise de Charles Morazé ${ }^{45}$.

\section{De la question du développement à celle des travailleurs immigrés}

Le Centre d'études des relations interethniques a notamment pour mission de promouvoir des études sur les conditions de succès des transferts de savoirs dans le cadre de la coopération. Sa direction est confiée à Pierre Bessaignet, également universitaire passé par l'UNESCO. Parmi les

43. Fischer G. et H. Laugier, op. cit., p. 24.

44. Meimon J., "L'invention de l'aide française au développement. Discours, instruments et pratiques d'une dynamique hégémonique ", op. cit.

45. Fischer G., « Henri Laugier : souvenirs. De l'ONU au Centre d'études des relations interethniques ", op. cit. 
hauts fonctionnaires promouvant les études sur les relations interethniques, il est le seul dont les travaux portent sur les minorités et les relations interethniques ${ }^{46}$. Par ailleurs, Pierre Bessaignet a eu un engagement politique bien distinct de ses trois collaborateurs. Il milite de 1945 à 1952 au sein de la Gauche communiste de France et, de 1945 à 1951, il figure avec Pierre Naville, Charles Bettelheim, Gilles Martinet et Maurice Nadeau au comité de rédaction de la Revue Internationale, revue généraliste non partisane et marxiste.

Sa carrière académique commence en 1940 aux États-Unis, où il suit une formation en ethnologie et en économie aux universités d'Harvard et de Yale et se spécialise en ethnologie économique. Outre ses travaux sur le capitalisme d'État en Suède ${ }^{47}$, certaines de ses recherches, bien avant 1966, portent sur le fait minoritaire, travaux développés notamment à l'occasion de son séjour aux États-Unis ou de ses missions menées pour l'UNESCO ${ }^{48}$. Dans le cadre de l'institution onusienne, il crée un département de sociologie à l'Université de Dacca au Bangladesh en 1956. En 1959, une autre mission le conduit avec Fredrik Barth à l'université de Téhéran, où il organise une section de recherches rurales. En 1961, il prend la direction du Centre de recherche de l'UNESCO sur le développement économique et social à Delhi. Si Pierre Bessaignet n'a pas eu de responsabilité à l'IEDES, il s'est ainsi très largement investi dans la politique de coopération scientifique : au Bangladesh, en Inde et en Iran, il a pour mission d'œuvrer au développement des sciences sociales. En 1966, il est nommé professeur titulaire de la chaire d'ethnologie à l'université de Nice, où il contribue à la mise en place du département de sociologie et d'ethnologie parallèlement à la direction du Centre d'études sur les relations interethniques.

Dirigé par un universitaire spécialiste des relations interethniques s'étant investi dans la politique scientifique de l'UNESCO, le Centre d'études s'inscrit directement dans la lignée du programme de recherche initié sur la scène internationale, réapproprié par des hauts fonctionnaires dans le cadre des transformations des relations extérieures de la France avec ses anciennes colonies. Les activités du Centre participent clairement à poser dans un même cadre de réflexion les enjeux de l’immigration et ceux de la coopération.

En 1972 et 1974, la Commission nationale et le Centre organisent deux colloques qui rassemblent hauts fonctionnaires et chercheurs ${ }^{49}$. Celui

46. Biographie établie à partir de : «Dossier documentaire ", Fonds Charles Morazé : 8D2 31 ; Collectif, Bulletin de l'IDERIC, n 5-6, 1988 ; Gaillard G., Dictionnaire des ethnologues et des anthropologues, Paris, Armand Colin, 1997, p. 195.

47. Bessaignet P., Coopération et capitalisme d'État : l'expérience suédoise de coopération agricole, Paris, PUF, 1953.

48. Bessaignet P., Tribesmen of the Chittagong Hill Tracts, Dacca, Museum buildings, 1958.

49. CERIN, La formation des coopérants, Paris, IDERIC, 1973 ; IDERIC, Les effets de la formation sur les travailleurs immigrés, IDRIC-Etudes préliminaires, Nice, 1975. 
d'avril 1972 porte sur la «formation des coopérants ». L'avant-propos de l'ouvrage tiré du colloque indique clairement la relation causale établie entre politique de développement et enjeux interculturels et interethniques. «L'assistance technique » aurait permis de constater que les contacts de l'expert/coopérant dans les pays d'accueil posaient des « problèmes de caractère interethnique, interculturel 50 ». Le colloque est censé répondre à une interrogation réflexive des organismes de formation, suscitée par la critique du modèle d'éducation français comme modèle absolu ${ }^{51}$. C'est pourquoi sont réunis avant tout des acteurs français de la politique de formation des coopérants et plus généralement de la politique de coopération ${ }^{52}$. La rencontre doit permettre de dresser un bilan des actions mises en ouvre et appelées à être améliorées en s'adaptant aux « milieux » pour favoriser des activités de coopération plus justes et plus efficaces, le « milieu » désignant l'environnement culturel. Les cadres autochtones formés sont perçus comme porteurs de référents culturels spécifiques et socialisés dans un environnement culturel et institutionnel distinct des coopérants. La prise en compte de ces référents culturels et de cet environnement doit être au fondement d'une formation adaptée, celle-ci reposant sur la mobilisation de savoirs spécifiques. En juin 1974, le colloque sur les « Travailleurs étrangers en Europe occidentale » est introduit par une allocution du secrétaire général de la Commission qui après avoir décrit les difficultés caractérisant la vie des travailleurs étrangers en Europe, conclut en invoquant la nécessité de contribuer au développement des pays d'émigration ${ }^{53}$. C'est bien le "paradigme développementaliste 54 » que l'on retrouve ici, liant régulation de l'immigration et celle du développement. Ce paradigme s'incarne à travers les formations qu'assure le Centre d'études via le Centre associé de formation aux relations interethniques (CAFRI) dirigé par le sociologue Michel Oriol et rassemblant d'autres sociologues. Il a pour mission de « mettre à la disposition de tous ceux qui, par leur activité professionnelle ou leur engagement, sont impliqués dans des relations interculturelles (militants syndicaux, enseignants, formateurs, travailleurs sociaux, coopérants, etc. $\left.{ }^{55}\right) »$.

50. CNEI (dir.), La formation des coopérants, Paris, IDERIC, 1973, p. 2.

51. Lettre d'invitation signée par Georges Fisher et datée du 8 octobre 1971. Fonds Charles Morazé : 8D2 23.

52. Notamment le Centre de formation des experts de la coopération technique internationale, le Centre national d'étude d'agronomie tropicale, l'Institut international de recherche et de formation «Éducation et développement », le Centre des hautes études administratives sur l'Afrique et l'Asie moderne, le Service de coopération technique de l'OCDE, la Division de la Recherche du Centre de Développement de l'OCDE ou encore le Centre associé de formation aux relations interethniques, centre de formation du Centre d'études sur les relations interethniques.

53. Bernard P.J. (dir.), Les travailleurs étrangers en Europe occidentale : actes, Paris, IDERIC, 1976, p. 18.

54. Dedieu J.-P., La parole immigrée : les migrants africains dans l'espace public en France, 19601995, op. cit.

55. Fonds Charles Morazé. 8D2 23. 
La genèse de la Commission nationale pour les études des relations interethniques par le prisme des trajectoires de ses principaux promoteurs permet d'identifier les contours d'un consensus au sein de l'administration scientifique : l'articulation des enjeux de l'immigration et de la coopération à travers leur problématisation en termes de relations interethniques et interculturelles semble devenue évidente. Cette représentation des enjeux, qui renvoie à une réévaluation de la position de la France sur la scène internationale alors qu'elle a perdu ses colonies, est partagée et défendue par des hauts fonctionnaires aux ancrages politiques pourtant distincts et dont les réseaux de sociabilités politiques et intellectuels ne se recoupent pas entièrement. C'est bien la socialisation dans les institutions onusiennes et dans le cadre des politiques de coopération scientifique internationale et nationale qui les réunit autour de la promotion des études sur les relations interethniques.

La reconstitution des trajectoires de ces hauts fonctionnaires, entre les institutions onusiennes et la politique scientifique française, permet de contribuer à l'histoire de la sociologie française des relations interethniques d'une part, et à celle du regard porté par l'administration sur les travailleurs immigrés d'autre part. Décentrer l'attention vers la construction d'un besoin de savoirs et la genèse d'un champ de recherche, autrement dit vers des acteurs a priori mineurs et en retrait par rapport à la politique de l'immigration mais centraux dans la politique scientifique, permet d'identifier des espaces de l'administration qui, s'ils sont restés dans l'ombre, documentent et éclairent le cadrage de la question immigrée et soulignent encore une fois la diversité des acteurs qui y participent. Bien que restés confidentiels, la Commission nationale et le Centre d'études ont ainsi permis l'introduction en France d'une problématisation de la question immigrée, autorisant une formulation recourant explicitement à la dimension culturelle des groupes sociaux - et ce, malgré le cadre légal de la politique de l'immigration, et grâce à l'appropriation par ces hauts fonctionnaires des réflexions onusiennes sur « la question des races" reformulées selon leur perception des intérêts de la France sur la scène internationale.

Avec les transformations de l'administration de l'immigration et les reconversions de hauts fonctionnaires des milieux de la coopération vers celui de l'immigration ${ }^{56}$, la genèse de la CNEI permet de mieux saisir comment la politique d'insertion interculturelle a pu prendre forme à la fin des années 1970, laissant intactes les catégories de désignation des immigrés du point de vue du cadre légal mais réintroduisant peu à peu la dimension culturelle au cœur de la question immigrée.

56. Laurens S., Une politisation feutrée. Les hauts fonctionnaires et l'immigration en France, Paris, Belin, 2009. 